\title{
Can APACHE II, SOFA, ISS, and RTS Severity Scores be Used to Predict Septic Complications in Multiple Trauma Patients?
}

\author{
Sebastian Trancă ${ }^{1}$, Cristina Petrișor ${ }^{1}$, Natalia Hagău${ }^{1}$, Constantin Ciuce ${ }^{2}$ \\ 1 Department of Anaesthesia and Intensive Care II, "Iuliu Hatieganu" University of Medicine and Pharmacy Cluj- \\ Napoca, Romania \\ 2 Surgical Department I, "Iuliu Hatieganu” University of Medicine and Pharmacy Cluj-Napoca, Romania
}

\begin{abstract}
Background: Physiological composite scores are used to predict mortality in multiple trauma patients. Sepsis is the leading cause of late mortality in trauma victims brought about by immune suppression due to homeostasis dysregulation.

Objective: To determine whether APACHE II, SOFA, ISS and RTS scores can predict the occurrence of sepsis in multiple trauma patients.

Methods: APACHE II, SOFA, ISS, and RTS scores were calculated during the first twenty-four hours after the injury for sixty-four adult poly-traumatic patients. The occurrence of infectious complications was investigated over a fourteenday period. The infection-free rates for the multiple trauma patients were considered as end-points in the KaplanMeier plot analysis.

Results: For SOFA, a cutoff score of 4 points was identified as a predictor of the occurrence of sepsis, with $89 \%$ of the patients with SOFA $<4$ being infection-free, while $37 \%$ of those with SOFA $>4$ were infection-free $(p<0.01)$. None of the patients with APACHE II $\leq 5$ points developed infections. Eighty-four percent of patients with APACHE II scores of 5-10 did not develop sepsis, while $49 \%$ with APACHE II $\geq 11$ were infection-free $(p<0.01)$. A cutoff of 7 points was found to be most discriminative for RTS. Eighty-eight percent of the patients with RTS $\geq 7$ and $43 \%$ of those with RTS $<7$ were infection-free $(p<0.01)$. Eighty-eight percent of patients with ISS $<22$ did not develop sepsis and $56 \%$ with ISS $\geq 22$ did not develop sepsis $(p<0.01)$.

Conclusion: APACHE II, SOFA, ISS, and RTS functional severity scores can predict mortality as well as the occurrence of sepsis in multiple trauma patients.
\end{abstract}

Keywords: trauma, sepsis, intensive care unit

Received: 24 April 2016 / Accepted: 01 July 2016

\section{INTRODUCTION}

Multiple trauma patients have a high risk of developing sepsis. Thus early diagnosis and the commencement of immediate antibiotic treatment are mandatory to reduce morbidity and mortality [1].

The differentiation between the trauma-induced systemic inflammatory response syndrome (SIRS) and the occurrence of sepsis in multiple trauma victims is not always straightforward as both present with similar clinical and laboratory findings [2,3]. Moreover, the results of bacteriological cultures taken to identify mi- croorganisms potentially responsible for sepsis are often delayed and may yield both false negative and false positive results when colonization cannot be differentiated from infection [4].

Sepsis is the leading cause of late mortality in trauma victims and is due to immune suppression caused by homeostasis dysregulation. Physiological composite scores are used to predict mortality in multiple trauma patients. The Glasgow Coma Scale, the mean blood pressure, and the need to provide mechanical ventilation, are calculated from the clinical variables. If the clinical and physiological variables demonstrate good 
predictive abilities, especially when combined with functional severity scores, they could be used to evaluate the risk of in-hospital mortality as well as the risk of developing severe infections in multiple trauma victims.

The objective of this study was to determine whether the composite functional severity scores, ISS (The Injury Severity Scores), RTS (The Revised Trauma Score), APACHE II (The Acute Physiology and Chronic health Evaluation II) and SOFA (The Sequential Organ Failure Assessment) could predict the occurrence of sepsis in multiple trauma patients.

\section{- METHODS}

\section{Subjects}

The enrollment of patients into this prospective observational study was approved by the Ethics Committee of the Iuliu Hațieganu University of Medicine and Pharmacy Cluj-Napoca (Approval no. 549/2012). All included patients, or the next of kin, gave written informed consent for patients to participate and records to be used in the study. Sixty-four multiple trauma patients, aged over 18 years, having an Injury Severity Score (ISS) higher than 16 points, who survived at least twelve hours after a traumatic injury and who were admitted to the Intensive Care Unit (ICU) of the Clinical Emergency County Hospital of Cluj between November 2012- November 2013, were admitted to the study.

Exclusion criteria were: burn injuries, chronic renal insufficiency, thyroid diseases, chronic liver diseases, autoimmune diseases, and previous treatment with immunosuppressive drugs.

For each patient, demographic data, clinical findings, laboratory results and physiological parameters were recorded upon admission. In the first twentyfour hours post trauma the following functional severity scores were calculated: ISS (The Injury Severity Scores), RTS (The Revised Trauma Score), APACHE II (The Acute Physiology and Chronic health Evaluation II), SOFA (The Sequential Organ Failure Assessment), as well as GCS (Glasgow Coma Scale).

Any clinical suspicion of infection was confirmed by positive cultures obtained from biological samples (urine, tracheal aspirates, blood cultures). A diagnosis of pneumonia was made if new infiltrates were observed on chest X-rays, together with positive cultures.
A diagnosis of a severe systemic bloodstream infection was made based on the identification of recognized pathogens in blood cultures. Urinary tract infections were confirmed based on urinary cultures in excess of $10^{5}$ microorganisms $/ \mathrm{mL}$. Surgical site infections were confirmed by the identification of organisms from aseptically obtained specimens collected from the skin and subcutaneous tissue associated with any of the surgical incisions. Each patient was considered to be infection-free at fourteen days if no infection could be identified (Group T) or a patient who presented infectious complications (Group T\&S) when one or more septic complications were diagnosed. The occurrences of infectious complications were followed up for fourteen days after the traumatic event, and were considered as end-points.

Blood samples were taken, and patients' treatment included complex haemodynamic and respiratory monitoring, volemic resuscitation, continuous norepinephrine infusion if septic shock was present, organ support, empiric antimicrobial therapy until the bacteriological results were available, and guided antibiotic therapy thereafter, nutrition, stress ulcer prophylaxis and deep vein thrombosis prophylaxis, multimodal analgesia and blood transfusions if required, together with physical therapy.

\section{Statistical analysis}

Unpaired t-test assuming equal variances to compare mean values for continuous data and Chi-squared test with Yates' correction for categorical data was used. The level of significance was set at alpha $=0.05$.

Kaplan Meier plots were used to analysis the time lapse from admission to the time when the infection was diagnosed [5].

The percentage of patients who were infection-free at 14 days was the considered the end-point. The discriminators were the cutoffs identified as optimal thresholds for each clinical parameter or composite functional severity scores. The thresholds were determined by correlating the clinical parameter or the severity scores ISS, RTS, SOFA, and APACHE II, with the outcome: patient with infection or patient free of infection at 14 days, by using Cox proportional hazard models. The percentage of patients who developed infections was calculated for the groups with mean blood pressure, need for mechanical ventilation, ISS, RTS, SOFA, APACHE II, and GCS higher and lower than the optimal identified cutoff. 
126 The Journal of Critical Care Medicine 2016;2(3)

\section{RESULTS}

From the sixty-four multiple-trauma patients that were included in the study, eighteen (28.12\%) developed infections in the first fourteen days following a severe traumatic event (Table 1). Seventeen of these presented with pneumonia, five presented with urinary tract infections, one presented with surgical site infections, while six presented with bloodstream infections. There were no significant differences regarding age and gender distribution between patients who did not present with infectious complications (Group T)
Available online at: www.jccm.ro

and those who presented with infections during the study period.

According to clinical and laboratory data, prehospital hypotension and preexisting chronic diseases, as well as hypoxemia on admission, were associated with higher infection rates. The functional severity scores, GCS, SOFA, APACHE II, RTS, and ISS, at time of admission to the ICU, were more severely altered in the patients who developed infections later on, compared to those who did not $(\mathrm{p}<0.05)$ (Table 1$)$. Thus, the T\&S group patients presented a greater degree of physiological impairments on admission.

Table 1. Baseline data for patients with trauma who did not develop sepsis (T) and patients with trauma who developed sepsis during their Intensive Care Unit admission (T\&S)

\begin{tabular}{|c|c|c|c|c|}
\hline & & $\mathbf{T}$ & T\&S & p-value \\
\hline No & & 46 & 18 & \\
\hline age & & $47 \pm 16$ & $47 \pm 17$ & 0.844 \\
\hline \multirow{2}{*}{ Sex } & $\mathrm{F}$ & 11 & 6 & \multirow{2}{*}{$p=0.65$} \\
\hline & $\mathrm{M}$ & 35 & 12 & \\
\hline \multirow{2}{*}{ ISS } & $<22$ & 22 & 3 & \multirow{2}{*}{$p=0.02$} \\
\hline & $\geq 22$ & 24 & 15 & \\
\hline \multirow{3}{*}{ APACHE II } & $<5$ & 15 & 0 & \multirow{3}{*}{$p=0.02$} \\
\hline & $5-10$ & 11 & 2 & \\
\hline & $11+$ & 20 & 16 & \\
\hline \multirow{2}{*}{ RTS } & $<7$ & 16 & 14 & \multirow{2}{*}{$p<0.01$} \\
\hline & $\geq 7$ & 30 & 4 & \\
\hline \multirow{2}{*}{ GCS } & $<8$ & 7 & 10 & \multirow{2}{*}{$p<0.01$} \\
\hline & $\geq 8$ & 39 & 8 & \\
\hline Massive transfusion & \multirow{5}{*}{ no. } & 4 & 5 & $p=0.12$ \\
\hline Prehospital hypotension & & 8 & 13 & $p<0.01$ \\
\hline Preexisting chronic disease & & 12 & 10 & $p=0.03$ \\
\hline SIRS & & 38 & 17 & $p=0.41$ \\
\hline MOF & & 18 & 17 & $p<0.01$ \\
\hline SOFA & \multirow{7}{*}{ mean value } & $2.9(3.8)$ & $6(3)$ & $<0.001$ \\
\hline MAP (mmHg) & & $71.9(15.4)$ & $71(18)$ & 0.930 \\
\hline $\mathrm{pH}$ & & $7.32(0.1)$ & $7.27(0.1)$ & 0.05 \\
\hline $\mathrm{PaO} 2 / \mathrm{FiO} 2$ & & $330.3(145)$ & $240.8(84)$ & 0.004 \\
\hline Serum lactate (mg/dL) & & $30.1(22.4)$ & $36.5(21)$ & 0.158 \\
\hline Leukocytes (no./dL) & & $12710(5474)$ & $15188(6514)$ & 0.164 \\
\hline Blood glucose ( mg/dL) & & $158(67)$ & $195(85)$ & 0.103 \\
\hline \multirow{2}{*}{$\begin{array}{l}\text { Mechanical ventilation } \\
\text { days }\end{array}$} & $<3$ & 37 & 0 & \multirow{2}{*}{$p<0.01$} \\
\hline & $\geq 3$ & 9 & 18 & \\
\hline \multirow{2}{*}{ Trauma mechanism } & blunt & 37 & 10 & \multirow{2}{*}{$p=0.09$} \\
\hline & penetrating & 9 & 8 & \\
\hline
\end{tabular}


Table 2. Mean (SD) values for the severity scores in the first 24 hours after a traumatic event

\begin{tabular}{lccc} 
Severity score & $\mathbf{T}$ & T\&S & $\mathbf{p}$ \\
ISS & $25 \pm 10$ & $35 \pm 12$ & $<0.01$ \\
\hline RTS & $6.8 \pm 1.4$ & $5.17 \pm 2$ & $<0.01$ \\
\hline APACHE II & $10 \pm 9.1$ & $18 \pm 7$ & $<0.01$ \\
\hline SOFA & $3 \pm 3.8$ & $6 \pm 3$ & $<0.01$ \\
\hline
\end{tabular}

The predictive role of clinical parameters for the occurrence of sepsis in multiple trauma patients

A cutoff of 8 points was identified for GCS, patients with a GCS higher than 8 points having an increased risk of developing sepsis compared to those with a GCS lower than 8 points ( $83 \%$ versus $19 \%, \mathrm{p}<0.01)$ (Figure 1 ).

Only 25\% patients who presented pre-admission hypotension (systolic blood pressure below $90 \mathrm{mmHg}$ ), were free of infectious complications, while $88 \%$ of the patients who did not present with prehospital hypotension were free from infections at 14 days $(\mathrm{p}<0.01)$ (Figure 1).

The initial need for mechanical ventilation was also investigated as a prognostic marker. Patients requiring mechanical ventilation were at higher risk of developing sepsis compared to those who were mechanically ventilated in the first seventy-two hours $(\mathrm{p}<0.01)$ (Figure 1). None of the patients requiring mechanical ventilation within the first three days, subsequent developed sepsis.

\section{Sepsis prediction based on the functional severity} scores in multiple trauma patients

Patients who developed infectious complications during the first fourteen days after a traumatic event had much altered initial functional scores compared to those who did not (Table 2).

For the SOFA score, by using Kaplan-Meier plot analysis, an optimal cutoff value of 4 points was identified as being the most discriminative. Eighty-nine percent of the patients with a SOFA lower than 4 did not present with infections during the study period, while $37 \%$ of patients with SOFA $>4$ did not present with an infection. $(\mathrm{p}<0.01)$ (Figure $2 \mathrm{~A})$.

None of the patients with an APACHE II score lower than 5 points developed sepsis. During the study period, $84 \%$ of patients with an APACHE II scores ranging from 5 to 10 points did not develop infections, while only $49 \%$ of patients with an APACHE II score higher than 11 points did not present any infections $(\mathrm{p}<0.01)$ (Figure 2B).
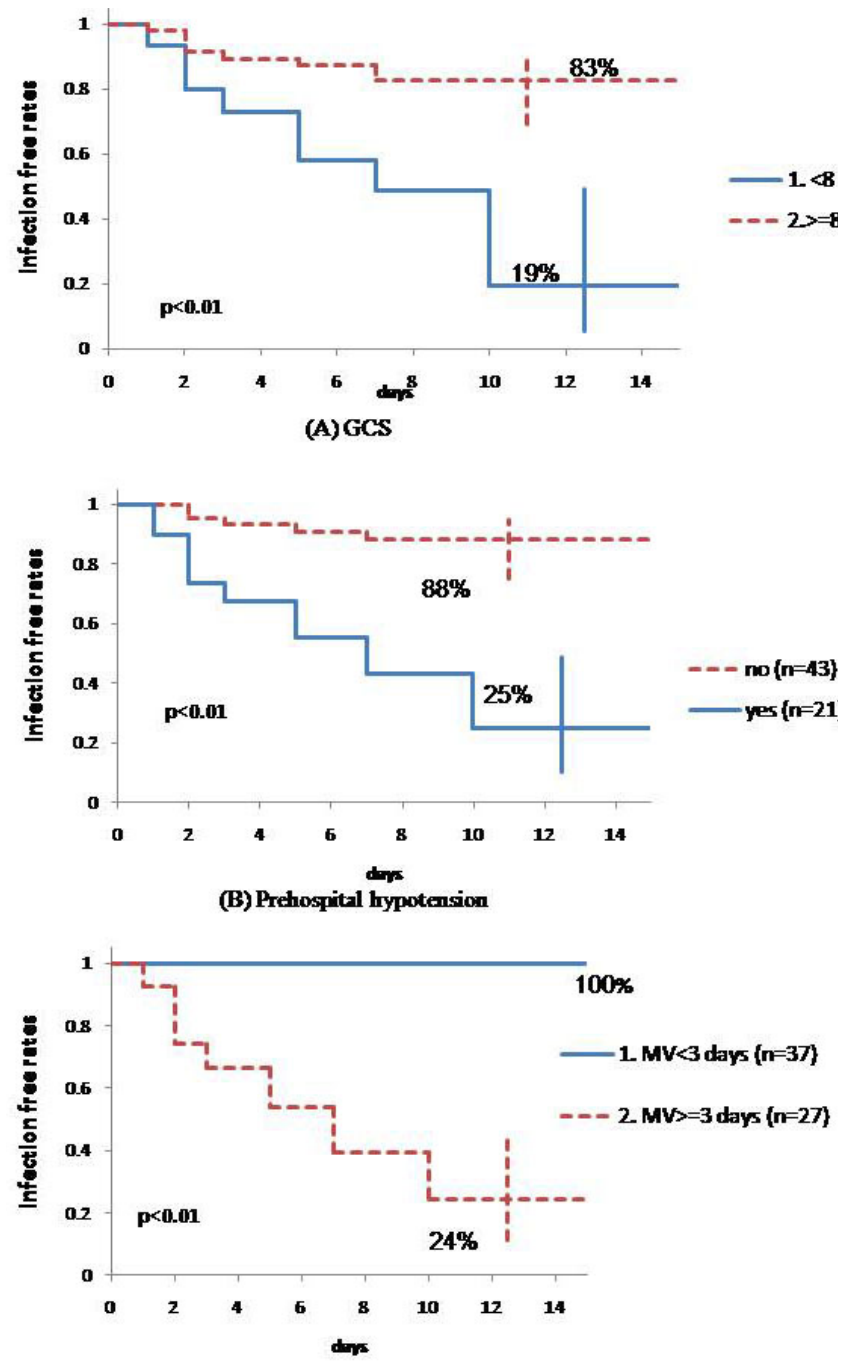

(C) Mechanical ventilation (MV)

Fig. 1. Kaplan-Meier plot analysis for the predictive role of clinical characteristics of the occurrence of sepsis in multiple trauma patients: (A) GCS higher and lower than 8 points; (B) prehospital hypotension (systolic blood pressure below $90 \mathrm{mmHg}$ ); and (C) the need for more than 72 hours of mechanical ventilation. Infection-free rates= the percentage of patients without a positive diagnosis of infection at 14 days.

For the RTS, an optimal cutoff of 7 points was identified. Eighty-eight percent of patients with an RTS higher than 7 points did not develop infections, while $43 \%$ of the patients with RTS lower than 7 points did not develop infections $(\mathrm{p}<0.01)$ (Figure $2 \mathrm{C})$.

For ISS, an optimal threshold of 22 points was identified as the most discriminative. Eighty-eight percent of patients with an ISS lower than 22 points were free of infections at fourteen days, while $56 \%$ of those with ISS higher than 22 did not develop sepsis $(\mathrm{p}<0.01)$ (Figure 2D). 

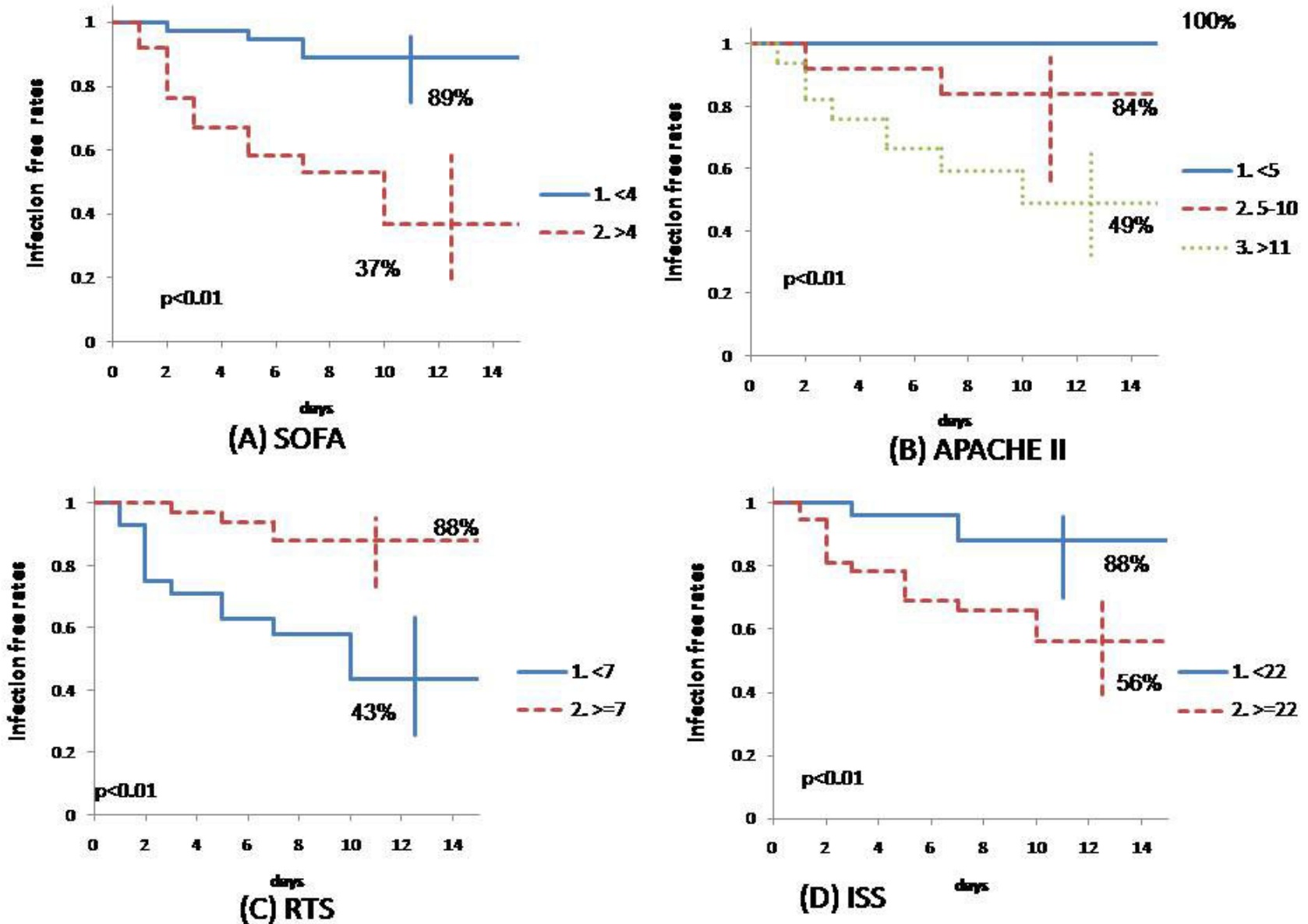

Fig. 2. Kaplan-Meier analysis for severity scores' predictive role for the occurrence of sepsis in multiple trauma patients. (A) SOFA score higher and lower than 4 points; (B) APACHE II scores below 5 points, ranging 5-10 points, or higher than 11 points; (C) RTS lower and higher than 7 points; and (D) ISS higher and lower than 22 points. Infection-free rates= the percentage of patients without a positive diagnosis of infection at 14 days.

\section{DIsCUSSION}

The significance of SIRS (systemic inflammatory response syndrome) in the diagnosis of sepsis is seriously threatened by the new definitions of sepsis [5]. This response aims to restore homeostasis and represents the core immunopathological alterations that induce multiple organ dysfunctions, and can also be caused by severe traumatic lesions. The processes that characterize the recently discarded SIRS concept, i.e. activation of the immune cell function, induction of stress hormones synthesis, synthesis of arachidonic acid derivatives, activation of the coagulation cascade and the complement system, all play important physiological roles.

These proinflammatory responses may lead to multiple organ dysfunctions with possibly lethal consequences [6-8]. The severity of the initial injury characterizing each system and organ can be quantified.
The initial severity of an injury is reflected in the clinical state of the patient and can be measured by using physiological parameters. For nervous system dysfunction, the Glasgow Coma Scale serves to assess motor activity. Cardio-vascular function is quantified initially by measuring blood pressure, heart rate, and seric lactate. Respiratory dysfunction is reflected in breathing rate and blood gasses, alterations in which might imply the need for mechanical ventilation. All the clinical and biological parameters, which are obtained by analyzing each organ and system, reflect the degree of severity of the initial injury. The joint use of these parameters has lead to complex functional severity scores, the validation of which, through large sample studies, have resulted in their routine use as outcome predictors. Some of these composite scores, such as the APACHE II and SOFA can be applied to all critically ill patients, while others, like ISS and RTS, are specific for trauma cases 
[9-14]. The usefulness of these scores is that they allow the calculation, and therefore the prediction, of the risk of death, as an outcome measure.

In multiple trauma patients, the initial proinflammatory response syndrome is soon replaced by an antiinflammatory response that leads to immune suppression and a higher risk of septic complications [15]. Late mortality following trauma is frequently caused by septic complications that are due to traumatic lesionsassociated immunosuppression [16,17].

Functional scores are used to assess mortality in critically ill patients who display organ dysfunctions on admission to an ICU. These scores have been used in practice for more than 50 years and have the advantage that they are easy to use, cheap and reliable, and have been already validated. The ICU physician may accurately predict the outcome regarding mortality by performing calculations based on clinical and laboratory data. Mortality rates have been investigated and quantified for each composite score.

Most frequently, in all ICU-patient categories, SOFA and APACHE II scores are calculated. These have also been applied to multiple trauma patients. In this category of patients, the mortality rates can also be evaluated based on specific scores, like RTS and ISS, which are used to complement the general scales [18].

The usefulness of these scores resides in the fact that they allow physicians to direct efforts towards patients who would benefit most following initial triage. They can facilitate organizational and management decisions by assessing the level of care given in each ICU and permit a comparison of different centers.

The clinical scoring systems may be capable of predicting a diverse set of outcomes [19]. In the current study, the hypothesis was that the functional composite scores, as they relate to mortality rates, may be predictors of sepsis in multiple trauma patients. Previously, it has been suggested that the predictive potential of the scoring systems was usually no better than clinical judgment [19]. The current study findings suggest that both the clinical characteristics and the composite scores may be used to predict the occurrence of sepsis in multiple trauma patients.

All the investigated composite scores discriminated between two categories of patients, those with scores higher and lower than the optimally identified thresholds. Patients with scores greater than the defined limits had an increased risk of developing sepsis, while patients with lower scores, presented lower infection rates. Practically, the more severe the initial injury, the more altered were the physiological conditions. The severity of the disease correlated well with the rates of occurrence of sepsis, possibly by inducing marked immune suppression.

The sample size of the reported study is modest, and being a single center study, generalization of the results is limited.

The results of this study could indicate that functional severity scores may be considered an additional tool to be used in the comparison of prognostic indicators in multiple trauma patients.

\section{CONCLUSIONS}

The functional severity scores APACHE II, SOFA, ISS, and RTS, predict not only the mortality rate but also the risks of occurrence of sepsis in multiple trauma patients.

\section{ACKNOWLEDGMENTS}

The authors thank all the medical personnel working in the Intensive Care Unit of the Clinical Emergency County Hospital Cluj for their clinical work and support and Mr. Nicolae Todor for the statistical advice.

\section{DISCLOSURE}

The authors have no conflicts of interests to declare.

\section{REFERENCES}

1. Balci C, Sungurtekin H, Gürses E, Sungurtekin U, Kaptanoglu B. Usefulness of procalcitonin for diagnosis of sepsis in the intensive care unit. Crit Care. 2003;7:85-90.

2. Adams CA. Sepsis Biomarkers in Polytrauma Patients. Crit Care Clin. 2011;27:345-54.

3. Kibe S, Adams K, Barlow G. Diagnostic and prognostic biomarkers of sepsis in critical care. J Antimicrob Chemother. 2011;66:33-40.

4. Castelli GP, Pognani C, Meisner M, Stuani A, Bellomi D, Sgarbi L. Procalcitonin and C-reactive protein during systemic inflammatory response syndrome, sepsis and organ dysfunction. Crit Care. 2004;8:R234-42.

5. Singer $M$, Deutschman CS, Seymour CW, et al. The Third International Consensus Definitions for Sepsis and Septic Shock (Sepsis-3). JAMA. 2016;315:801-10. 
130 - The Journal of Critical Care Medicine 2016;2(3)

6. Wojtaszek M, Staśkiewicz G, Torres K, Jakubowski K, Rácz O, Cipora E. Changes of procalcitonin level in multiple trauma patients. Anaesthesiol Intensive Ther. 2014;46:78-82.

7. Rajkumari N, Mathur P, Sharma S, Gupta B, Bhoi S, Misra MC. Procalcitonin as a predictor of sepsis and outcome in severe trauma patients: a prospective study. J Lab Physicians. 2013;5:100-8.

8. Angus DC, Wax RS. Epidemiology of sepsis: an update. Crit Care Med. 2001;29:S109-16.

9. Antonelli M, Moreno R, Vincent JL, et al. Application of SOFA score to trauma patients. Sequential Organ Failure Assessment. Intensive Care Med. 1999;25:389-94.

10. Mica L, Furrer E, Keel M, Trentz O. Predictive ability of the ISS, NISS, and APACHE II score for SIRS and sepsis in polytrauma patients. Eur J Trauma Emerg Surg. 2012;38:665-71.

11. Castelli GP, Pognani C, Cita M, Stuani A, Sgarbi L PR. Procalcitonin, C-reactive protein, white blood cells and SOFA score in ICU: diagnosis and monitoring of sepsis. Minerva Anestesiol. 2006;72:69-80.

12. Agarwal A, Agrawal A, Maheshwari R. Evaluation of Probability of Survival using APACHE II \& TRISS Method in Orthopaedic Polytrauma Patients in a Tertiary Care Centre. J Clin Diagn Res.
Available online at: www.jccm.ro

2015;9:RC01-4.

13. Brattström O, Granath F, Rossi P, Oldner A. Early predictors of morbidity and mortality in trauma patients treated in the intensive care unit. Acta Anaesthesiol Scand. 2010;54:1007-17.

14. Keel M, Eid K, Labler L, Seifert B, Trentz O, Ertel W. Influence of Injury Pattern on Incidence and Severity of Posttraumatic Inflammatory Complications in Severely Injured Patients. European Journal of Trauma. 2006;32:387-95.

15. Lenz A, Franklin GA, Cheadle WG. Systemic inflammation after trauma. Injury. 2007;38:1336-45.

16. Lausević Z, Vuković G, Stojimirović B, Trbojević-Stanković J, Resanović V, Lausevic M. Kinetics of C-reactive protein, interleukin-6 and -10, and phospholipase A2-II in severely traumatized septic patients. Vojnosanit Pregl. 2010;67:893-7.

17. Pierrakos C, Vincent J-L. Sepsis biomarkers: a review. Crit Care. 2010;14:R15.

18. Bouillon B, Lefering R, Vorweg M, Tiling T, Neugebauer E, Troidl H. Trauma score systems: Cologne Validation Study. J Trauma. 1997;42:652-8.

19. Gortzis LG, Sakellaropoulos F, Ilias I, Stamoulis K, Dimopoulou I. Predicting ICU survival: a meta-level approach. BMC Health Serv Res. 2008;8:157. 